\title{
Higher Dimensional Schwinger-like Anomalous Effective Action *
}

\author{
A.Smailagic ${ }^{\dagger}$ \\ Department of Physics, Faculty of Electrical Engineering \\ University of Osijek, Croatia \\ E. Spallucci ${ }^{\ddagger}$ \\ Dipartimento di Fisica Teorica \\ Università di Trieste, \\ INFN, Sezione di Trieste
}

\begin{abstract}
We construct explicit form of the anomalous effective action, in arbitrary even dimension, for Abelian vector and axial gauge fields coupled to Dirac fermions. It turns out to be a surprisingly simple extension of 2D Schwinger model effective action.
\end{abstract}

\footnotetext{
*Accepted for publication on Phys.Rev.D

†E-mail address: a.smailagic@etfos.hr

‡E-mail address:spallucci@trieste.infn.it
} 
Problem of anomalies in field theory is an outstanding and old one. Since the early days of their discovery [1], [2], anomalies have been approached and investigated in many ways 円. Only in two spacetime (2D) dimensions the corresponding anomalous effective action was calculated thanks to a particularly simple, anomalous Feynman diagram. In this way famous Schwinger model and Liouville gravity anomalous actions were found [3]. Extending the calculation of the effective action from $2 \mathrm{D}$ to $4 \mathrm{D}$ one is faced with considerable technical difficulties for the explicit calculation of the finite, i.e. non-local part of the anomalous triangle diagram. On the other hand, it is the non-local part of the effective action [1], [4], which is unambiguous and is the source of the anomalous behavior [5], [6]. Knowledge of the effective action, apart from giving a complete information on the theory, has proven crucial in the recent study of the two-dimensional gauge and gravitational anomalous theories in the light-cone gauge which has lead to important developments and understanding of this theory as related to strings [7].Surprisingly enough, though gravity is more complicated than YangMills gauge theory, it turned out that the 4D gravitational effective action could have been derived starting from the trace anomaly itself [8]. On the other hand, to our best knowledge, apart from mentioned 2D examples, the form of the 4D or higher dimensional anomalous gauge effective action is not present in the literature. Fortunately, difficulty related to the calculation of the effective action have not hindered study of anomalies since methods of anomaly calculation have been designed in such a way to avoid explicit knowledge of the effective action, so that divergences of the anomalous currents, were extracted from local counter-terms, [2], [9], [10].

In this paper, we would like to present the complete, anomalous, effective action for an

\footnotetext{
${ }^{1}$ Due to proliferation of literature on this subject, we are not able to quote all papers containing important contributions. We apologize to the authors of unquoted papers.

${ }^{2}$ We have given a reference of one of a comprehensive reviews on the two-dimensional models.
} 
Abelian gauge field theory coupled to Dirac fermions in even dimensions $D=2 k$.

To properly pave our way let us briefly recall the origin of gauge anomalies. Dirac fermions coupled to external gauge fields can be integrated out in a path integral and give the anomalous gauge field effective action induced by quantum effects. We choose to depart from a standard treatment of axial anomaly where chiral symmetry is a global invariance of the theory. Namely, fermions will posses local axial symmetry, so we introduce both vector and axial vector gauge fields in the covariant derivative. This approach is crucial in order to be able to construct effective action from which axial and vector current can be recovered.

Guidelines for construction of anomalous effective action are potentially anomalous Feynman diagrams, which in $2 k$ dimensions, have $k+1$ fermion propagators [1] with $2 m+1$ axial vertices and $k-2 m$ vector gauge field vertices. The odd number of vertices with axial vector gauge field follow from the condition $\left(\gamma^{5}\right)^{2 m+1}=\gamma^{5}$ and therefore such diagrams have the same internal structure as those with one axial vertex. On the basis of the above reasoning we found the general form of the anomalous gauge effective action in $D=2 k$ dimensions to be

$$
\begin{array}{r}
\Gamma=\sum_{m=0}^{m_{\max }} \frac{g^{2 m+1} e^{k-2 m}}{(2 \pi)^{k}} \int d^{2 k} x \epsilon^{\mu_{1} \ldots \mu_{2 k-4 m} \nu_{1} \ldots \nu_{4 m}} F_{\mu_{3} \mu_{4}} \ldots \times F_{\mu_{2 k-4 m-1} \mu_{2 k-4 m}} \times \\
F_{\nu_{1} \nu_{2}}^{5} \times \ldots \times F_{\nu_{4 m-1} \nu_{4 m}}^{5}\left[F_{\mu_{1} \mu_{2}} \frac{1}{\square}\left(\partial^{\mu} A_{\mu}^{5}\right)-2 \mathbf{a} A_{\mu_{1}} A_{\mu_{2}}^{5}\right]
\end{array}
$$

Short explanation is needed for the meaning of $m_{\max }$. The value of the integer $m$ is restricted by $m \leq(k-1) / 2$, thus $m_{\max }$ represents the maximal integer obtained from such relation.

From general arguments, as well as from explicit calculations, one expects that the extraction of anomalies is subject to regularization arbitrariness. This regularization dependence is seen in (11) through the presence of an arbitrary parameter a in the local part of the effective action [12]. The axial gauge current obtained from (11) is

$$
\begin{aligned}
& J^{5 \mu} \equiv \frac{1}{g} \frac{\delta S}{\delta A_{\mu}^{5}} \\
& =-\sum_{m=0}^{m_{\max }} \frac{g^{2 m} e^{k-2 m}}{(2 \pi)^{k}}\left[\epsilon^{\mu_{1} \ldots \mu_{2 k-4 m} \nu_{1} \ldots \nu_{4 m}} \frac{\partial^{\mu}}{\square}\left(F_{\mu_{1} \mu_{2}} \times \ldots \times F_{\mu_{2 k-4 m-1} \mu_{2 k-4 m}} F_{\nu_{1} \nu_{2}}^{5} \times \ldots \times F_{\nu_{4 m-1} \nu_{4 m}}^{5}\right)\right.
\end{aligned}
$$




$$
\begin{aligned}
& -4 m \epsilon^{\mu \mu_{2} \ldots \mu_{2 k-4 m+2} \nu_{3} \nu_{4} \ldots \nu_{4 m}} F_{\mu_{3} \mu_{4}} \times \ldots \times F_{\mu_{2 k-4 m+1} \mu_{2 k-4 m+2}} F_{\nu_{3} \nu_{4}}^{5} \times \ldots \times F_{\nu_{4 m-1} \nu_{4 m}}^{5} \frac{\partial_{\mu_{2}}}{\square} \partial A^{5} \\
& +\epsilon^{\mu \mu_{2} \ldots \mu_{2 k-4 m} \nu_{1} \ldots \nu_{4 m}} F_{\mu_{3} \mu_{4}} \times \ldots \times F_{\mu_{2 k-4 m-1} \mu_{2 k-4 m}} F_{\nu_{3} \nu_{4}}^{5} \times \ldots \times F_{\nu_{4 m-1} \nu_{4 m}}^{5} \times \\
& \left.\times\left(8 \mathbf{a} m F_{\nu_{1} \nu_{2}} A_{\mu_{2}}^{5}+2 \mathbf{a} m(1-4 m) F_{\nu_{1} \nu_{2}}^{5} A_{\mu_{2}}\right)\right]
\end{aligned}
$$

from (2) one finds anomalous divergence of the axial currents as

$$
\begin{array}{r}
\partial_{\mu} J^{5 \mu}=(\mathbf{a}-1) \sum_{m=0}^{m_{\max }} \frac{g^{2 m} e^{k-2 m}}{(2 \pi)^{k}} \epsilon^{\mu_{1} \ldots \mu_{2 k-4 m} \nu_{1} \ldots \nu_{4 m}} F_{\mu_{1} \mu_{2}} \times \ldots \times \\
F_{\mu_{2 k-4 m-1} \mu_{2 k-4 m}} F_{\nu_{1} \nu_{2}}^{5} \times \ldots \times F_{\nu_{4 m-1} \nu_{4 m}}^{5}
\end{array}
$$

On the other hand, the vector current obtained from (四) is

$$
\begin{aligned}
J^{\mu} \equiv & \frac{1}{e} \frac{\delta S}{\delta A_{\mu}}=\sum_{m=0}^{m_{\max }} \frac{g^{2 m+1} e^{k-2 m-1}}{(2 \pi)^{k}} \epsilon^{\mu_{1} \ldots \mu_{2 k-4 m} \nu_{1} \ldots \nu_{4 m}} F_{\mu_{5} \mu_{6}} \times \ldots \times F_{\mu_{2 k-4 m-1} \mu_{2 k-4 m}} \\
& \times F_{\nu_{1} \nu_{2}}^{5} \times \ldots \times F_{\nu_{4 m-1} \nu_{4 m}}^{5}\left[(k-2 m) F_{\mu_{3} \mu_{4}} \frac{\partial_{\mu_{2}}}{\square}\left(\partial^{\rho} A_{\rho}^{5}\right)\right. \\
& \left.-\mathbf{a}(k-2 m) F_{\mu_{3} \mu_{4}} A_{\mu_{2}}^{5}+\mathbf{a}(k-2 m-1) F_{\mu_{3} \mu_{4}}^{5} A_{\mu_{2}}\right]
\end{aligned}
$$

which leads to the vector gauge current anomalous divergence as

$$
\begin{aligned}
\partial_{\mu} J^{\mu}= & -\mathbf{a} \sum_{m=0}^{m_{\max }} \frac{g^{2 m+1} e^{k-2 m-1}}{(2 \pi)^{k}} \epsilon^{\mu_{1} \ldots \mu_{2 k-4 m} \nu_{1} \ldots \nu_{4 m}} F_{\mu_{1} \mu_{2}}^{5} F_{\mu_{3} \mu_{4}} \times \ldots \times \\
& \times F_{\mu_{2 k-4 m-1} \mu_{2 k-4 m}} F_{\nu_{1} \nu_{2}}^{5} \times \ldots \times F_{\nu_{4 m-1} \nu_{4 m}}^{5}
\end{aligned}
$$

In the above result one recognizes a well known pattern of incompatibility of classical symmetries, i.e. no choice of the parameter a can preserve both symmetries at the quantum level. To be truthful, it is the divergence of the axial current which normally appears in the literature [2], [11]. From the above result it is clear why, i.e. all explicit method of calculation assume gauge invariant regularization which in our language implies the choice $\mathbf{a}=0$. Furthermore, previous considerations were limited to global axial symmetry which amounts to setting all axial gauge fields couplings to zero. We strongly suggest the use of the parameter $\mathbf{a}$ in the formulae, and only a posteriori choose a particular value, i.e. a definite regularization scheme. In this way one can easily track the interplay and incompatibility of the classical symmetries at the quantum level. Furthermore, it permits easy shift from one 
local symmetry to another, no matter how complicated the expressions for currents are.

In order to make the general formalism more transparent, as well as to verify the agreement with known results, let us start from 2D. From (1), choosing $k=1$, one finds effective action as

$$
S=\frac{e g}{2 \pi} \int d^{2} x \epsilon^{\mu \nu}\left[F_{\mu \nu} \frac{1}{\square}\left(\partial^{\rho} A_{\rho}^{5}\right)-2 \mathbf{a} A_{\mu} A_{\nu}^{5}\right]
$$

Exploiting a 2D identity $A_{\mu}=\epsilon_{\mu \nu} A^{5 \nu}(e=g)$, transforms (6) in the well known form of the Schwinger effective action

$$
S=-\frac{e^{2}}{4 \pi} \int d^{2} x\left[F_{\mu \nu} \frac{1}{\square} F^{\mu \nu}+2 \mathbf{a} A^{\mu} A_{\mu}\right]
$$

together with the anomalous divergences of vector and axial vector current

$$
\begin{aligned}
& \partial_{\mu} J^{\mu}=-\mathbf{a} \frac{e}{\pi} \epsilon^{\mu \nu} \partial_{\mu} A_{\nu}^{5}=-\mathbf{a} \frac{e}{\pi} \partial_{\mu} A^{\mu} \\
& \partial_{\mu} J^{5 \mu}=(\mathbf{a}-1) \frac{e}{\pi} \epsilon^{\mu \nu} \partial_{\mu} A_{\nu}
\end{aligned}
$$

To proceed a step further, we take $k=2$ in (迎) and find, to our best knowledge, so far unreported 4D effective anomalous action

$$
S=\frac{g e^{2}}{4 \pi^{2}} \int d^{4} x \epsilon^{\mu \nu \alpha \beta}\left[F_{\mu \nu} F_{\alpha \beta} \frac{1}{\square}\left(\partial^{\rho} A_{\rho}^{5}\right)-2 \mathbf{a} A_{\mu} A_{\nu}^{5} F_{\alpha \beta}\right]
$$

which enables us to find explicit form of the vector and axial vector currents as

$$
\begin{aligned}
& J^{\mu}=\frac{e g}{4 \pi^{2}} \epsilon^{\mu \nu \alpha \beta}\left[F_{\alpha \beta} \frac{\partial_{\nu}}{\square}\left(\partial^{\rho} A_{\rho}^{5}\right)-2 \mathbf{a} F_{\alpha \beta} A_{\nu}^{5}+\mathbf{a} F_{\alpha \beta}^{5} A_{\nu}\right] \\
& J^{5 \mu}=-\frac{e^{2}}{4 \pi^{2}} \epsilon^{\rho \nu \alpha \beta}\left[\frac{\partial^{\mu}}{\square}\left(F_{\rho \nu} F_{\alpha \beta}\right)+2 \mathbf{a} \delta_{\mu}^{\rho} F_{\alpha \beta} A_{\nu}\right]
\end{aligned}
$$

In spite of the complicated form of currents (11) and (12), one finds simple looking, well known, 4D divergences

$$
\begin{aligned}
& \partial_{\mu} J^{\mu}=-e g \frac{\mathbf{a}}{4 \pi^{2}} \epsilon^{\mu \nu \alpha \beta} F_{\mu \nu} F_{\alpha \beta}^{5} \\
& \partial_{\mu} J^{5 \mu}=e^{2} \frac{(\mathbf{a}-1)}{4 \pi^{2}} \epsilon^{\mu \nu \alpha \beta} F_{\mu \nu} F_{\alpha \beta}
\end{aligned}
$$


We shall also exhibit a $6 \mathrm{D}(k=3)$ results because it is the first dimension in which more than one axial vertex can be present. In fact, one finds $m=0$ (one axial vertex), $m=1$ (three axial vertices). They give the anomalous action as

$$
\Gamma=\frac{g e}{(2 \pi)^{3}} \int d^{6} x \epsilon^{\mu \nu \rho \sigma \tau \omega}\left(e^{2} F_{\rho \sigma} F_{\tau \omega}+g^{2} F_{\rho \sigma}^{5} F_{\tau \omega}^{5}\right)\left[F_{\mu \nu} \frac{1}{\square} \partial A^{5}-2 \mathbf{a} A_{\mu} A_{\nu}^{5}\right]
$$

(15) leads to the anomalous divergences of currents as

$$
\begin{gathered}
\partial_{\mu} J^{\mu}=-g \frac{\mathbf{a}}{(2 \pi)^{3}} \epsilon^{\mu \nu \rho \sigma \tau \omega} F_{\mu \nu}^{5}\left[e^{2} F_{\rho \sigma} F_{\tau \omega}+g^{2} F_{\rho \sigma}^{5} F_{\tau \omega}^{5}\right] \\
\partial_{\mu} J^{5 \mu}=e \frac{\mathbf{a}-1}{(2 \pi)^{3}} \epsilon^{\mu \nu \rho \sigma \tau \omega} F_{\mu \nu}\left[e^{2} F_{\rho \sigma} F_{\tau \omega}+g^{2} F_{\rho \sigma}^{5} F_{\tau \omega}^{5}\right]
\end{gathered}
$$

In (16) and (17) we see the appearance of axial vertices, in particular the difference in the axial current conservation compared to [1]. The presence of additional $F_{\rho \sigma}^{5}$ is caused by the local axial symmetry considered in this paper.

In summary, we have constructed anomalous effective action for Abelian gauge fields which represents higher dimensional extension of 2D Schwinger model. To our satisfaction, the anomalous effective action turns out to have particularly simple form whose kernel is a $2 \mathrm{D}$ effective action written in terms of independent vector and axial vector gauge fields. The higher dimensional input is all encoded in the sequence of vector and axial vector gauge field strengths which contract remaining indices in the $2 k$-dimensional Levi-Civita tensor. 


\section{REFERENCES}

[1] S.L. Adler, Phys.Rev.177, 2426, (1969)

[2] S.E.Treiman, R. Jackiw, D.J.Gross "Lectures on Current Algebra and its Applications" , Princeton UP, Princeton NJ, (1972)

[3] T.Berger " Fermions in two (1+1)-dimensional anomalous gauge theories: the chiral Schwinger model and the chiral quantum gravity " Hamburg U. DESY-90-084, July 1990.

[4] L.Rosenberg, Phys.Rev. 129, (1963) 2786

[5] R.Jackiw " Topological Investigations of Quantized Gauge Theories " in Relativity, Groups and Topology eds. B.deWitt and R.Stora (Elsevier, Amsterdam 1984)

[6] M.T.Grisaru, N.K.Nielsen, W.Siegel, D.Zanon Nucl.Phys.B247, 157 (1984)

[7] A.M. Polyakov, Phys.Lett. B103, 207, (1981); A.M. Polyakov, Mod. Phys.Lett. A2, 893, (1987)

[8] R .J. Riegert Phys.Lett. 134B 56, (1984)

[9] K.Fujikawa Phys.Rev.Lett. 42, 1195, (1979)

[10] B.deWitt, Relativity, Groups and Topology, Paris (1963);

A.O.Barvinsky, G.A.Vilkovisky, Phys.Rep. 119, 1, (1985)

[11] P.H.Frampton, T.W.Kephart Phys.Rev.Lett. 50, 1343, (1983);

L. Alvarez-Gaume, E.Witten, Nucl.Phys.234, 269 (1983)

[12] A.Smailagic, R.E.Gamboa-Saravi Phys.Lett. 192B 145, (1987);

A.Smailagic, E.Spallucci Phys.Lett. 284B 17, (1992) 